Anaesthesist 2015 - 64:641-642

DOI 10.1007/s00101-015-0083-x

Online publiziert: 2. September 2015

(c) Springer-Verlag Berlin Heidelberg 2015

\section{F. Bloos}

Klinik für Anästhesiologie und Intensivmedizin, Center for Sepsis Control \& Care (CSCC),

Universitätsklinikum Jena, Jena, Deutschland

\title{
Behandlung invasiver Mykosen
}

\section{Was tun, wenn die Therapie versagt?}

Patienten, die eine intensivmedizinische Versorgung benötigen, tragen ein hohes Risiko für invasive Candida-Infektionen. Bei kritisch kranken Patienten liegen gleich mehrere Risikofaktoren vor, die diese Patienten für eine invasive CandidaInfektion prädestinieren. Auf der anderen Seite wird bei nichtneutropenischen Patienten mit neuen Infektionen initial nicht notwendigerweise die Hinzunahme einer antimykotischen Therapie zusätzlich zur empirischen antibakteriellen Therapie empfohlen [2]. Üblicherweise ist die Indikation für eine antimykotische Therapie an den mikrobiellen Nachweis von Pilzen geknüpft. Die European Society of Clinical Microbiology and Infectious Diseases hat 2012 diesbezüglich ihre Empfehlungen zu Diagnose und Therapie von Candida-Infektionen publiziert [3]. In erster Linie konzentriert sich die Leitlinie auf die Diagnose und die Einleitung einer adäquaten Therapie der invasiven Pilzinfektionen. In der Tat ist die Indikationsstellung einer solchen Behandlung gerade bei Intensivpatienten komplex genug. Eine Vielzahl von Übersichtsbeiträgen widmet sich dann auch in Anlehnung an die Leitlinien gerade diesem Thema. Dennoch wird selbst bei sorgfältiger Indikationsstellung die antimykotische Therapie nicht immer zu einer suffizienten Behandlung der Infektion führen. Daher erscheint es notwendig, zu einem bestimmten Zeitpunkt in der Behandlung die Adäquatheit einer antimykotischen Therapie infrage $\mathrm{zu}$ stellen und das therapeutische Vorgehen entsprechend zu adjustieren. In der Literatur finden sich in dieser Hinsicht jedoch nur wenige Hinweise für das optimale Vorgehen. Ist ein solches Überdenken der Behandlungsstrategie bei Pilzinfektionen daher weniger re- levant für die behandelnden Ärzte auf Intensivstationen?

\section{Dilemma in der Behandlung der invasiven Candida-Mykose}

Der Goldstandard für die Initiierung einer systemischen antimykotischen Therapie ist der Nachweis von Candida-Spezies in der Blutkultur oder in primär sterilen Körperflüssigkeiten wie z. B. Aszites oder Pleurapunktat [3]. Eine solche zielgerichtete Therapie soll bis 14 Tage nach der ersten negativen Blutkultur fortgeführt werden. Dieses Vorgehen beinhaltet jedoch ein zeitliches Problem, da der Kulturnachweis von Candida-Spezies mehrere Tage in Anspruch nehmen kann und das Sterberisiko für den Patienten mit einer unbehandelten invasiven Candida-Infektion täglich steigt [5]. Aus diesem Grund nennt die Leitlinie noch andere Therapiestrategien, die das Zeitproblem umgehen sollen [3]. Dazu gehören die prophylaktische Therapie (Behandlung von Patienten mit Risikofaktoren für eine invasive Candida-Infektion ohne Infektionszeichen), die empirische Therapie (Behandlung von Patienten mit Risikofaktoren für eine invasive Candida-Infektion mit Infektionszeichen) und die präemptive Therapie (Behandlung bei frühen diagnostischen Tests, die hinweisend auf invasive Candida-Infektionen sind, wie z. B. Candida-Score oder Serum- $(1,3)-\beta$ D-Glukan-Konzentration). Die Datenlage für diese Strategien ist jedoch noch unzureichend; es ist insbesondere unklar, wie Patienten, die von einer antimykotischen Therapie profitieren könnten, genau identifiziert werden sollen.

Dies bedeutet letztendlich, dass der Intensivmediziner entweder nur bei einer hohen Diagnosesicherheit behandelt, dafür aber einen Zeitverzug in der Einleitung einer antimykotischen Therapie in Kauf nimmt, oder aber auf Verdacht behandelt, dabei jedoch häufig übertherapiert. In diesem Spannungsfeld überrascht es nicht, dass auf Intensivstationen Antimykotika häufig ohne Nachweis einer Pilzinfektion und oft nicht leitlinienkonform gegeben werden [1]. Gerade im Licht der schwierigen und nicht selten unsicheren Indikationsstellung für Antimykotika bei kritisch kranken Patienten muss ins Kalkül gezogen werden, dass die initiale antimykotische Therapie u. U. nicht die korrekte Behandlung darstellt.

\section{Therapieversagen - was nun?}

In den Leitlinien für die Behandlung bakterieller Infektionen - wie z. B. den S3Leitlinien zur Behandlung nosokomialer Pneumonien [4] - ist der Umgang mit dem Therapieversagen der initialen antimikrobiellen Behandlung mit entsprechenden differenzialdiagnostischen Überlegungen genau umrissen. In diesem Sinne sollten wir auf unseren Intensivstationen nicht nur Strategien für die Initiierung einer antimykotischen Therapie implementieren, sondern auch Vorgehensweisen im Umgang mit einem Therapieversagen niedergelegen. In der Publikation von Arens et al. in dieser Ausgabe von Der Anaesthesist werden entsprechende Strategien dargelegt und ausführlich diskutiert. Dabei liegt der Schwerpunkt auf den invasiven Candida-Infektionen als die häufigsten Pilzinfektionen auf Intensivstationen, doch auch Infektionen mit Schimmelpilzen finden Beachtung. 


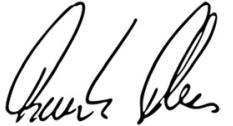

F. Bloos

\section{Korrespondenzadresse}

PD Dr. F. Bloos Ph.D.

Klinik für Anästhesiologie und Intensivmedizin, Center for Sepsis Control \& Care (CSCC)

Universitätsklinikum Jena

Erlanger Allee 101, 07747 Jena

Frank.Bloos@med.uni-jena.de

Interessenkonflikt. F. Bloos gibt an, dass kein Interessenkonflikt besteht.

\section{Literatur}

1. Azoulay E, Dupont H, Tabah A et al (2012) Systemic antifungal therapy in critically ill patients without invasive fungal infection*. Crit Care Med 40:813822. doi:10.1097/CCM.0b013e318236f297

2. Brunkhorst FM, Reinhart K (2009) [Diagnosis and causal treatment of sepsis]. Internist 50:810-816. doi:10.1007/s00108-008-2287-5

3. Cornely OA, Bassetti M, Calandra Tet al (2012) ESCMID* guideline for the diagnosis and management of Candida diseases 2012: non-neutropenic adult patients. Clin Microbiol Infect 18(Suppl 7):19-37. doi:10.1111/1469-0691.12039

4. Dalhoff K, Abele-Horn M, Andreas S et al (2012) Epidemiologie, Diagnostik und Therapie erwachsener Patienten mit nosokomialer Pneumonie. Pneumologie 66:707-765. doi:10.1055/s-0032-1325924

5. Kollef M, Micek S, Hampton N et al (2012) Septic shock attributed to Candida infection: importance of empiric therapy and source control. Clin Infect Dis 54:1739-1746. doi:10.1093/cid/cis305
Berghold, F., Brugger, H., Burtscher et al. (Hrsg.)

\section{Alpin- und Höhenmedizin}

Wien: Springer 2015, 485 S., 124 Abb., (ISBN 978-3-7091-1832-0), Hardcover, 68.00 EUR

Historisch gesehen hat die breite Palette der Alpinmedizin zwei markante Wurzeln: aus dem alpinen Rettungswesen, das ursprünglich mit dem Abtransport von toten Unfallopfern ins Tal beschäftigt war, entwickelte sich um die Mitte des vorigen Jahrhunderts, vor rund 60 Jahren, die Disziplin der alpinen Notfallmedizin, Hand in Hand mit der Rettung aus der Luft. Der zweite bedeutsame Grundpfeiler heißt Mount Everest, der nach jahrzehntelangem erfolglosen Bemühen schließlich 1953 erstbestiegen wurde, und zwar mit entscheidender Unterstützung der damals erst jungen, kaum etablierten wissenschaftlichen Disziplin der Höhenmedizin. Innerhalb der modernen Alpinmedizin gibt es heute zwei Themenbereiche, die aufgrund ihrer Besonderheiten ausschließlich der Alpinmedizin „gehören": Die Lawinenmedizin und die Medizin der großen und extremen Höhen. Die übrige Themenfülle, mit der sich das vorliegende Buch befasst, ist eine breite Palette von spannenden alpinmedizinischen Inhalten.

Vor diesem Hintergrund präsentiert dieses Buch einen faszinierenden Überblick über die aktuelle Alpin- und Höhenmedizin.

Im Gegensatz zum üblichen, oft geradezu manischen Bemühen um fachliche Konformität eines Kompendiums wurden die Autoren dieses Buches immer wieder dazu ermutigt, in ihrem ganz persönlichen Stil zu schreiben, was sie für richtig halten - und nicht, was „man" für die Wahrheit hält. Das ist lebendig, das ist so spannend wie die Wirklichkeit. Um der breiten Thematik gerecht zu werden haben fast 40 Autoren, darunter viele bekannte Exponenten der deutschsprachigen Alpinund Höhenmedizin, aber auch Kollegen aus Frankreich, Italien und Spanien an diesem Standardwerk mitgearbeitet. Dieses Buch durchzieht thematisch ein „roter Faden“ durch die drei Abschnitte 1. Alpine Sportmedizin, 2. Alpine Unfallmedizin und 3. Höhenmedizin: Der Bogen beginnt mit den sehr unterschiedlichen alpinistischen Belastungsmustern, der Leistungssteigerung und energetischen Taktik, was auch spezifische Ernährungsfragen aufwirft. Neben höhenbedingten- gibt es auch gesundheitliche Einschränkungen internistischer oder orthopädischer Natur. Besondere Empfehlungen gelten für Kinder und Schwangere, aber auch für die junge Disziplin des Sportkletterns.

Der zweite Abschnitt Alpine Unfallmedizin beginnt mit dem Risikomanagement und der Prävention von Bergunfällen, bevor die Besonderheiten und Herausforderungen, die Möglichkeiten und Grenzen der Erstversorgung Verunglückter im oft extrem schwierigen alpinen Gelände behandelt werden. Blitzschäden, Kälteschäden und Lawinenunfälle zählen zu den typischen alpinen Unfällen, aber auch internistische Notfälle im Gebirge und die logistischen Sonderfälle Canyoningund Höhlenunfall gehören dazu. Besondere ärztliche Einsatztaktiken, die Technik der lebensrettenden Sofortbergung und die medizinische Ausrüstung sind ebenso Höhepunkte dieses Buches wie die Anforderungskriterien des modernen Berg- und Flugrettungsarztes. Der dritte Abschnitt Höhenmedizin befasst sich mit den Phänomenen in verschiedenen Höhenlagen, wo die Luft lebensbedrohlich dünn ist, und mit der Anpassungsfähigkeit des Menschen, mit den Ursachen, der Klinik und Therapie der Höhenkrankheit. Der dritte Abschnitt beschreibt die vielschichtigen Aspekte der medizinischen Ausrüstung und ärztlichen Betreuung beim Höhentrekking und Höhenbergsteigen. Nicht zuletzt öffnet der Gesundheitstourismus in alpinen Höhen ganz neue Einsichten und Möglichkeiten.

Fazit

Das Herausgeber- und Autorenteam besteht aus dem who-is-who der Europäischen Alpinund Höhenmedizin; dementsprechend ist der Inhalt des Buches hervorragend gelungen. Farbige Abbildungen hätten mir noch besser gefallen, die aber den Preis des Buches sicher erhöht hätten.

Prof. Dr. V. Wenzel, Innsbruck 